\title{
Corrigendum
}

\section{Childhood interleukin-6, C-reactive protein and atopic disorders as risk factors for hypomanic symptoms in young adulthood: a longitudinal birth cohort study}

\author{
J. F. Hayes, G. M. Khandaker, J. Anderson, D. Mackay, S. Zammit, G. Lewis, D. J. Smith and \\ D. P. J. Osborn
}

https://doi.org./10.1017/S0033291716001574, Published online by Cambridge University Press 1 August 2016.

The authors would like to apologise for a numerical mistake in the abstract and table 3 of the above mentioned article.

In the abstract of the article, paragraph 'After adjusting for age, sex, ethnicity, socio-economic status, past psychological and behavioural problems, body mass index and maternal postnatal depression, participants in the top third of IL-6 values at 9 years, compared with the bottom third, had an increased risk of hypomanic symptoms by age 22 years [adjusted odds ratio 1.77, 95\% confidence interval (CI) 1.10-2.85, p<0.001]. Higher IL-6 levels in childhood were associated with adult hypomania features in a dose-response fashion. After further adjustment for depression at the age of 18 years this association remained (adjusted odds ratio 1.70, 95\% CI 1.03-2.81, $\mathrm{p}=$ 0.038). There was no evidence of an association of hypomanic symptoms with CRP levels, asthma or eczema in childhood'.

Should read:

'After adjusting for age, sex, ethnicity, socio-economic status, past psychological and behavioural problems, body mass index and maternal postnatal depression, participants in the top third of IL- 6 values at 9 years, compared with the bottom third, had an increased risk of hypomanic symptoms by age 22 years [adjusted odds ratio 1.77, $95 \%$ confidence interval (CI) 1.10-2.85, p = 0.018]. Higher IL-6 levels in childhood were associated with adult hypomania features in a dose-response fashion. After further adjustment for depression at the age of 18 years this association remained (adjusted odds ratio 1.70, 95\% CI 1.03-2.81, $\mathrm{p}=0.038$ ). There was no evidence of an association of hypomanic symptoms with CRP levels, asthma or eczema in childhood'. 
Table 3

Table 3. Serum IL-6 and CRP tertiles at age 9 years and the odds of hypomania aged 22 years

\begin{tabular}{|c|c|c|c|c|c|c|c|}
\hline & \multirow[b]{2}{*}{ Tertile } & \multirow[b]{2}{*}{$n$} & \multirow[b]{2}{*}{ Hypomania, $n(\%)$} & \multicolumn{4}{|c|}{ Hypomania symptoms: OR (95\% CI) $[p]$} \\
\hline & & & & Unadjusted & Model $1^{\mathrm{a}}$ & Model $2^{\mathrm{b}}$ & Model $3^{c}$ \\
\hline$n^{\mathrm{d}}$ & & & & 1717 & 1699 & 1201 & 7809 \\
\hline \multicolumn{8}{|l|}{ IL-6 } \\
\hline & Bottom & 573 & $30(5.24)$ & 1 & 1 & 1 & 1 \\
\hline & Middle & 551 & $39(7.08)$ & $1.38(0.84-2.25)[0.202]$ & $1.51(0.91-2.48)[0.104]$ & $1.59(0.91-2.79)[0.106]$ & $1.53(0.98-2.42)[0.064]$ \\
\hline & Top & 593 & $47(7.93)$ & $1.55(0.97-2.50)[0.069]$ & $1.78(1.09-2.89)[0.020]$ & $1.87(1.07-3.27)[0.028]$ & $1.77(1.10-2.85)[<0.001]$ \\
\hline & Linear trend & 1717 & $116(6.76)$ & $1.24(0.98-1.56)[0.069]$ & $1.32(1.04-1.67)[0.021]$ & $1.36(1.03-1.78)[0.027]$ & $1.31(1.04-1.65)[0.022]$ \\
\hline \multicolumn{8}{|l|}{ CRP } \\
\hline & Bottom & 622 & $52(8.36)$ & 1 & 1 & 1 & 1 \\
\hline & Middle & 532 & $34(6.39)$ & $0.75(0.48-1.17)[0.207]$ & $0.83(0.53-1.31)[0.428]$ & $0.87(0.52-1.48)$ [0.614] & $0.84(0.52-1.34)[0.480]$ \\
\hline & Top & 567 & $30(5.29)$ & $0.61(0.38-0.97)$ [0.038] & $0.69(0.43-1.11)[0.125]$ & $0.71(0.40-1.28)[0.232]$ & $1.04(0.67-1.62)[0.872]$ \\
\hline & Linear trend & 1721 & $116(6.74)$ & $0.78(0.62-0.98)[0.033]$ & $0.83(0.65-1.05)[0.128]$ & $0.85(0.64-1.13)[0.266]$ & $1.02(0.81-1.29)[0.877]$ \\
\hline
\end{tabular}

Should read:

Table 3. Serum IL-6 and CRP tertiles at age 9 years and the odds of hypomania aged 22 years

\begin{tabular}{|c|c|c|c|c|c|c|c|}
\hline & \multirow[b]{2}{*}{ Tertile } & \multirow[b]{2}{*}{$n$} & \multirow[b]{2}{*}{ Hypomania, $n(\%)$} & \multicolumn{4}{|c|}{ Hypomania symptoms: OR (95\% CI) [ $p]$} \\
\hline & & & & Unadjusted & Model $1^{a}$ & Model $2^{\mathrm{b}}$ & Model $3^{c}$ \\
\hline$n^{\mathrm{d}}$ & & & & 1717 & 1699 & 1201 & 7809 \\
\hline \multicolumn{8}{|l|}{ IL-6 } \\
\hline & Bottom & 573 & $30(5.24)$ & 1 & 1 & 1 & 1 \\
\hline & Middle & 551 & $39(7.08)$ & $1.38(0.84-2.25)[0.202]$ & $1.51(0.91-2.48)[0.104]$ & $1.59(0.91-2.79)[0.106]$ & $1.53(0.98-2.42)[0.064]$ \\
\hline & Top & 593 & 47 (7.93) & $1.55(0.97-2.50)$ [0.069] & $1.78(1.09-2.89)$ [0.020] & $1.87(1.07-3.27)$ [0.028] & $1.77(1.10-2.85)$ [0.018] \\
\hline & Linear trend & 1717 & $116(6.76)$ & $1.24(0.98-1.56)[0.069]$ & $1.32(1.04-1.67)[0.021]$ & $1.36(1.03-1.78)[0.027]$ & $1.31(1.04-1.65)[0.022]$ \\
\hline \multicolumn{8}{|l|}{ CRP } \\
\hline & Bottom & 622 & $52(8.36)$ & 1 & 1 & 1 & 1 \\
\hline & Middle & 532 & $34(6.39)$ & $0.75(0.48-1.17)[0.207]$ & $0.83(0.53-1.31)[0.428]$ & $0.87(0.52-1.48)[0.614]$ & $0.84(0.52-1.34)[0.480]$ \\
\hline & Top & 567 & $30(5.29)$ & $0.61(0.38-0.97)[0.038]$ & $0.69(0.43-1.11)[0.125]$ & $0.71(0.40-1.28)[0.232]$ & $1.04(0.67-1.62)[0.872]$ \\
\hline & Linear trend & 1721 & $116(6.74)$ & $0.78(0.62-0.98)[0.033]$ & $0.83(0.65-1.05)[0.128]$ & $0.85(0.64-1.13)[0.266]$ & $1.02(0.81-1.29)[0.877]$ \\
\hline
\end{tabular}


986 Corrigendum

\section{Reference}

Hayes JF, Khandaker GM, Anderson J, Mackay D, Zammit S, Lewis G, Smith DJ and Osborn DPJ (2016). 'Childhood interleukin-6, C-reactive protein and atopic disorders as risk factors for hypomanic symptoms in young adulthood: a longitudinal birth cohort study'. Psychological Medicine, 1-11. doi:10.1017/S0033291716001574 